\title{
609 COMBINING BINTRAFUSP ALFA WITH ABITUZUMAB ENHANCES SUPPRESSION OF THE TGF- $\beta$ SIGNALING PATHWAY
}

Feng Jiang ${ }^{*}$, Hong Wang, Tsz-Lun Yeung, Guozhong Qin, Bo Marelli, Hui Huang, KinMing Lo, Yan Lan. EMD Serono, Billerica, MA USA, Billerica, MA, USA

Background Bintrafusp alfa is a first-in-class bifunctional fusion protein composed of the extracellular domain of the TGF- $\beta$ RII receptor fused to a human IgG1 antibody blocking PDL1. The TGF- $\beta$ RII moiety of bintrafusp alfa functions as a "trap" to sequester active TGF- $\beta$ but does not block TGF- $\beta$ release from its latent form. Multiple mechanisms lead to the release of active TGF- $\beta$. Integrins control local activation of latent TGF- $\beta$ stored in the extracellular matrix and cell-surface reservoirs in the tumor microenvironment (TME). Alpha $\mathrm{v}$ integrin mRNA expression is correlated with multiple TGF- $\beta$ gene signatures. It has been shown that $\alpha v \beta 8$ integrin mediates TGF- $\beta$ activation without releasing it from the latent TGF- $\beta$ complex, suggesting that the TGF- $\beta$ RII moiety of bintrafusp alfa may be unable to sequester TGF- $\beta$ activated by $\alpha v \beta 8$ integrin. Therefore, we hypothesize that combining abituzumab, a pan- $\alpha v$ integrin antibody, with bintrafusp alfa may lead to enhanced suppression of TGF- $\beta$ signaling.

Methods The expression of $\alpha v$ and $\beta 6$ integrin mRNA was determined by RNA sequencing of triple-negative breast cancer (TNBC) tumor samples from a phase 1 clinical trial of bintrafusp alfa and correlated with patient response to bintrafusp alfa. The combination of bintrafusp alfa and abituzumab was investigated in vitro and in vivo in a TGF- $\beta$-dependent human tumor model, Detroit 562. In this study, CellTiter-Glo 2.0 Assay measured cell proliferation in vitro and enzymelinked immunosorbent assay measured the level of latencyassociated protein (LAP). A TGF- $\beta$ reporter cell line MDAMB-231 measured the level of active TGF- $\beta$. Antitumor activity in vivo was evaluated via tumor growth of Detroit 562 xenograft model in SCID mice.

Results In TNBC, increased expression of $\alpha \mathrm{v}$ and $\beta 6$ integrin mRNA was associated with poor response to bintrafusp alfa, suggesting that TGF- $\beta$ activated by $\alpha \mathrm{v}$ integrin may not be blocked by bintrafusp alfa. In Detroit 562 cells, abituzumab increased LAP levels in the cell culture medium, confirming modulation of the TGF- $\beta$ pathway. As a result, the amount of active TGF- $\beta$ released into culture medium was reduced by abituzumab. In vitro, both abituzumab and bintrafusp alfa suppressed Detroit 562 cell proliferation, and the combination suppressed cell proliferation further. In vivo, the combination led to increased tumor growth inhibition of Detroit 562 xenograft tumors relative to either monotherapy, further supporting the potential of this combination.

Conclusions Collectively, these preclinical findings support clinical development of bintrafusp alfa and abituzumab combination therapy to maximally suppress TGF- $\beta$ signaling in the TME.

Acknowledgements We thank George Locke for his analysis of the RNAseq data.

Ethics Approval This study was approved by the Institutional Animal Care and Use Committee at EMD Serono, Inc.; approval number [17-008].

http://dx.doi.org/10.1136/jitc-2021-SITC2021.609 